\title{
WSGC NASA Internship Final Report
}

\author{
Tessa Rundle ${ }^{1}$ \\ Department of Physics and Astronomy \\ Carthage College \\ Kenosha, WI
}

\begin{abstract}
This report describes the activities carried out during a 10-week, full-time internship at NASA's Johnson Space Center (JSC) in Houston, Texas. As an intern on the Space Suit Assembly (SSA) Development Engineering team, I contributed to several ongoing projects related to the advancement of Extravehicular Activity (EVA) space suits. Current EVA suits were designed for limited activities and usage time, and projected requirements of future exploration missions necessitate research into new suit components and materials. During my internship, I contributed mainly to the testing protocol for upcoming High-Performance EVA Glove (HPEG) testing, tasks for Z-2 suit testing, and studied the effects of radiation on space suit materials.
\end{abstract}

\section{Internship Overview}

The SSA team is a part of the Crew and Thermal Systems Division (CTSD) at JSC. The CTSD is responsible for the development of technology related to environmental control, life support systems, and thermal control systems for space craft and space suits. Within the CTSD, the SSA team is a part of the Space Suit and Crew Survival Systems Branch, which is responsible for managing several suit systems. The SSA team is focused on advancing EVA/exploration space suits.

As an intern with the SSA team, I attended weekly meetings to discuss current work, events, and testing relating to the team's projects and to update the team of my progress on what I had been working on. I also attended weekly tag-ups to discuss each project I was assigned with the corresponding lead or mentor of that project. The SSA team is currently focused on the development of the Z-2 space suit (Fig. 2), an advanced EVA suit with technologies that will eventually be used in a suit designed for Mars exploration. Current work on EVA suits includes improvements to overall mobility, the study of material resistance to radiation effects, and the introduction of suit docking mechanisms to reduce astronaut pre-breathe time and consumables, among other developments.

\section{Projects}

High-Performance EVA Glove (HPEG). During my internship, I worked on several tasks related to HPEG testing. HPEGs are being developed mainly for improvements in hand mobility and life span over current EVA glove designs (Walsh, 2015). HPEG testing will be taking place in fall 2016.

${ }^{1}$ Funding provided by the Wisconsin Space Grant Consortium through the National Space Grant College and Fellowship Program: NASA Training Grant \#NNX15AJ12H. Special thanks to my mentor, Amy Ross, and the suit team at JSC. 
My first task contributed to the selection of new test subjects for the upcoming testing. In order to be considered a good fit for testing, subjects must have hand sizes that correspond to one of two glove sizes, nominally "small" or "large". Hand measurements had been previously taken of 24 possible test subjects and three baseline subjects for the "small" glove size and three for the "large" size. Measurements included the circumference and length of the thumb and each finger of each subject's dominant hand, as well as the total hand length, palm width, and palm circumference. To determine which of the new subjects had the optimal hand size for testing, I needed to correlate their hand measurements to the measurements of the baseline "small" and "large" subjects.

To do this, I wrote a Python program to calculate the correlation coefficient between the possible test subject's hand measurements and the average of the measurements in each of the existing "small" and "large" pools. Correlation coefficients are used to determine the linear dependence between two variables or two sets of data (Weisstein). The correlation coefficient is a number between -1 and 1 . A correlation coefficient of 1 indicates total positive correlation, 0 indicates no correlation, and -1 indicates negative correlation.

The program successfully calculated the correlation between the measurements of each possible test subject and each of the two size pools, and sorted the results by the top size matches (highest correlation coefficient) in the "small" and "large" categories. The results will be used to recommend test subjects who are considered the best match for future glove testing.

Another task I completed for the HPEG project was updating the protocol for upcoming glovebox testing. Glovebox testing allows performance capabilities of space suit gloves to be evaluated at low pressures (Fig 1). The glovebox testing protocol includes various activities for the test subject to perform to analyze grip strength, pinch strength, tactility, dexterity, and more. Along with another intern, I worked to update the protocol's accuracy in accordance with the current test plan. In addition, fixtures and accessories to be used in the testing have been updated, so the protocol was also edited appropriately to account for these updates.

Updates were also made to the glove comfort report in the testing protocol. The purpose of the glove comfort report is to record various types of hand discomfort (touching, pressure points, pinching, thermal, etc.) that could be Fig. 1: A pair of space suit gloves being used in experienced by a subject during testing, and the severity the glovebox. During testing, various tools and and location of the discomfort. Previous versions of the protocol did not include all parts of the hand in the report, and only included general areas (i.e., "fingers" and "hand")

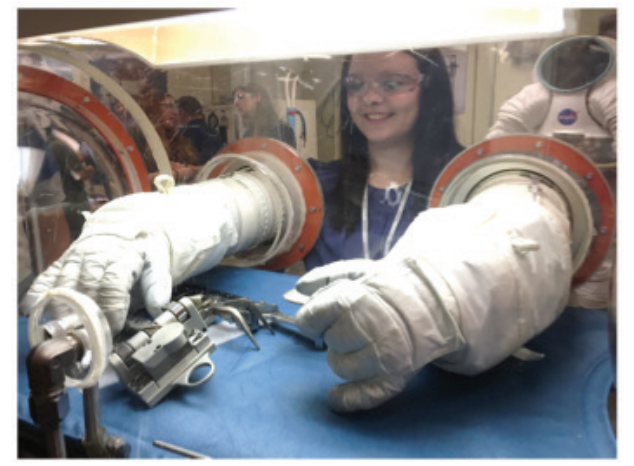
as places where discomfort could be experienced. The protocol was updated to include specific regions on both the palmar and dorsal sides of the hand and on each finger, as each type of discomfort could be experienced anywhere on the hand. 
Camera Mount Design for Z-2 Testing. The Z-2 suit is scheduled to be tested in the Neutral Buoyancy Lab (NBL) in fall 2016. In this testing, performance capabilities of the suit will be assessed as test subjects use tools to execute tasks similar to those performed during International Space Station EVAs. For these tests, a mockup of the Portable Life Support System (PLSS) will be attached to the Z-2 suit.

For the upcoming testing, I was assigned the task of designing a camera mount to be attached to the PLSS mockup. The camera used in testing is housed in a sealed case, and is used to provide a video feed of the test subject's workspace during the testing. I was provided with a camera case of the kind used at the NBL, as well as a list of requirements for the camera mount:

- Camera must be installed to the mount using no or minimal tools

- Must provide a "quick-release" (the camera case must be easily removed from the mount in case of an emergency)

- Must not obstruct or interfere with any of the test subject's motion or test activities

- Must be mounted in a location which provides a clear video view

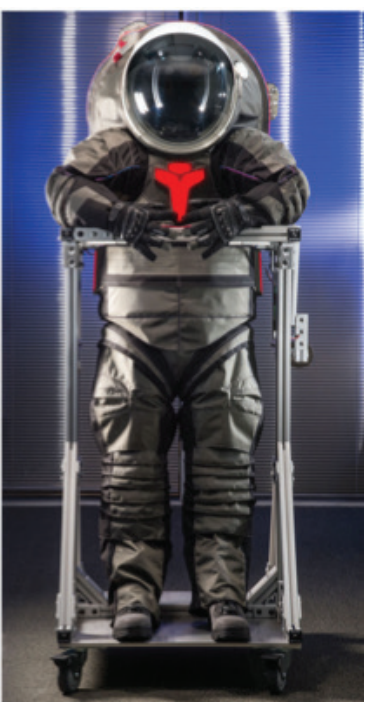
of the test subject's workspace

The camera mount design was created and updated using Creo Parametric design software. Design iterations were presented at NBL test meetings, and revised based on team and lab technician input. The final version of the camera mount, shown in (Fig. 3), was fabricated on-site at JSC in the Advanced Suit Lab.
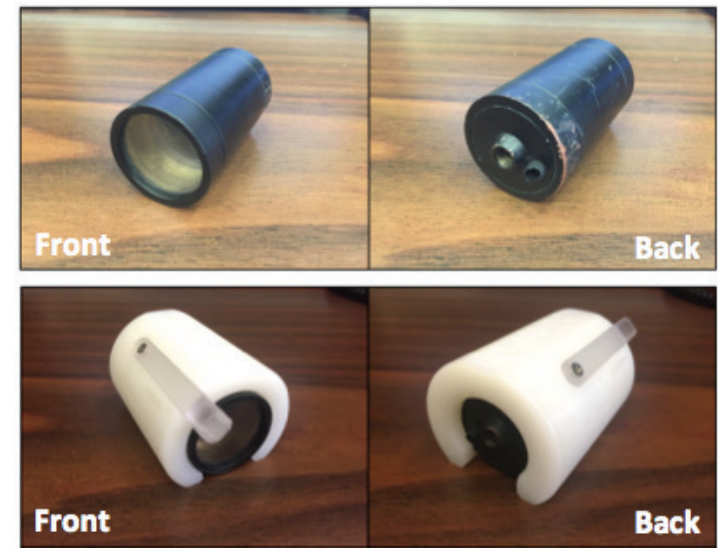

Fig. 3: Top: Camera case to be used in NBL testing. Bottom: Camera case inserted into camera mount that will be attached to the PLSS mockup for NBL testing. Design includes lever that can be depressed to allow for insertion of the camera case, but secures the case into the mount in normal position. No tools are required to insert or remove the case from the mount.

The camera mount will be integrated with the PLSS mockup in mid-August to prepare for NBL testing beginning shortly after. 
Irradiated Space Suit Materials Testing. A key challenge with proposed missions to Mars is the hazard of high-energy cosmic rays and other ionizing radiation. There is currently a lack of radiation damage data on space suit materials at doses that would be experienced on a mission to Mars, namely for solar particle event (SPE) proton exposures, high energy $(>=1 \mathrm{GeV})$ galactic cosmic ray (GCR) radiation, or a combination of SPE and GCR. SPE radiation is generated by solar flares or coronal mass ejections from the Sun, and consists of primarily directional protons with energies in the range of tens to hundreds of MeV. GCR radiation originates outside of the solar system and consists of primarily omnidirectional protons, along with silicon, iron, and other nucleons, present with particle energies on the order of $1 \mathrm{GeV}$ (Schimmerling, 2011).

In collaboration with White Sands Test Facility (WSTF), JSC is performing a study to quantify the effects of SPE and GCR radiation on space suit materials. If it is determined that properties of space suit materials change significantly when exposed to high levels of radiation, it is possible that space suits could fail on long duration missions to deep space, resulting in serious consequences for the mission.

This study focuses on three materials used or proposed as space suit restraint layers: Spectra, Dacron, and Vectran. In the spring of 2016 , a set of $20 \mathrm{~cm}$ x $20 \mathrm{~cm}$ samples of each fabric was sent to Brookhaven National Laboratory for irradiation.

The radiation doses for each sample were estimated by a JSC collaborator to correspond with the SPE and GCR radiation that would be experienced on a round trip to Mars, where most time is spent in deep space, with a significant amount of time spent on the Martian surface.

A previous SSA intern started work to perform baseline tensile testing on each of the three space suit materials per American Society for Testing and Materials (ASTM) standards. The method used for this testing was ASTM 5035 (Standard Test Method for Breaking Force and Elongation of Textile Fabrics - Strip Method), which utilizes 1.5 x 6" fabric strip samples. Each of the samples are then raveled (threads are individually removed) to create a 1 x 6" sample

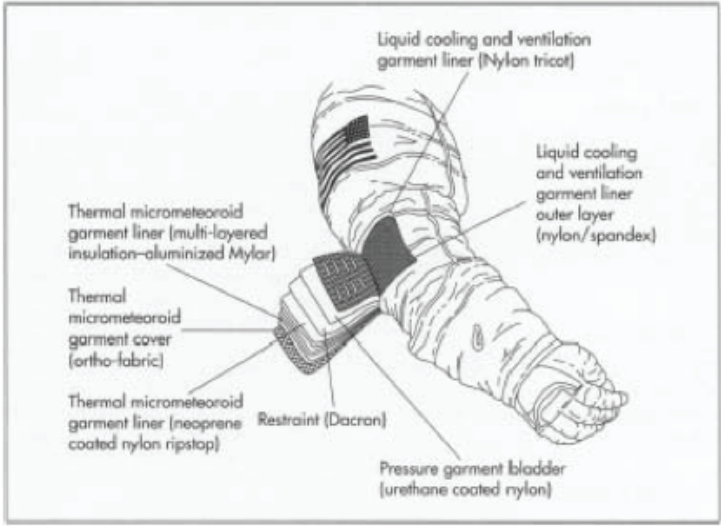

Fig. 4: Layers of the Extravehicular Mobility Unit (EMU) space suit, currently used in EVAs. The restraint layer, which is made of Dacron on the EMU, helps the suit maintain its shape when pressurized. Source: NASA. for testing. This test produces values for the breaking force and elongation of the specimen at the time of the break, and is done via an Instron tensile testing machine (Fig 6). Due to the high strength of the materials being tested, inconsistencies were observed with the breaking behavior of the test specimens. The specimens often broke close to the grips as opposed to the more ideal break location in the center of the specimen, the specimens slipped inside the grips causing a difference in gauge length for each test, and the application of tensile stress on the specimens (particularly Spectra) produced further unravelling of the samples (Fig. 5). Each of these inconsistencies contributed to a higher-than-desired standard deviation in the recorded values for breaking force and elongation. These inconsistencies also reduce the repeatability of the test. 
For this project, I needed to find a method that works to successfully tensile test irradiated and non-irradiated samples of Spectra, Dacron, and Vectran fabrics. As the most inconsistencies were observed with previous testing of Spectra, I decided to find a test that works well on Spectra, and then apply it to Dacron and Vectran. First, I completed a detailed review of all ASTM testing standards for textiles to determine which would be relevant for space suit materials. Because of the issues with the Spectra specimen unraveling during the ASTM 5035 strip test, I decided to try testing individual strands of Spectra as per ASTM 2256 (Standard Test Method for Tensile Properties of Yarns by the Single-Strand

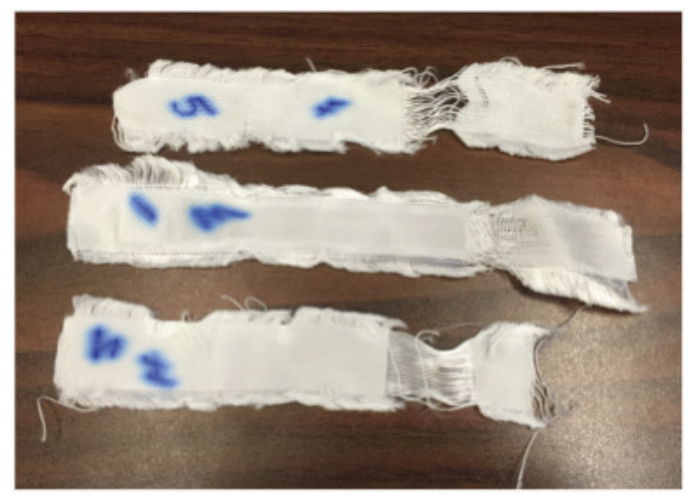

Fig. 5: 1 × 6" strips of Spectra fabric tested according to ASTM 5035 at JSC.

Method). As this method called for a minimum specimen length of $\sim 20$ ", some modifications to allow for $20 \mathrm{~cm}$ strand sizes were implemented. Spectra threads were sampled from both the warp and fill direction of the fabric roll, and tested in separate runs. In this round of testing, it was difficult to mount the short strands in the test grips and ensure an even pressure distribution on the upper and lower grips. The poor grip resulted in consistent specimen slippage throughout the testing, and significant differences in breaking force and elongation were observed.

While working on this project, I participated in regular update telecons with collaborators at WSTF, which were particularly useful for discussing test methods and receiving valuable advice and suggestions for further testing. From recommendations and research into standard measures to mitigate grip slippage during tensile testing, I decided to add cardboard tabs to the end of each strand, adhered with epoxy. A diagram of the new specimen configuration is shown in (Fig. 7). This modification is commonly used in tensile testing of single textile fibers, as well as in tensile testing of composite materials. The ASTM 2256 strand test was repeated using the modified specimens, and there were significant improvements in grip slippage.

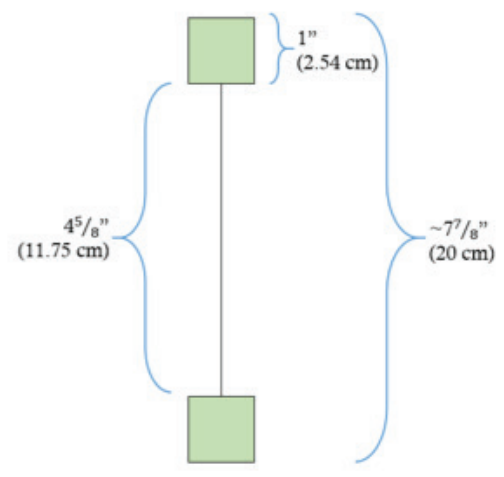

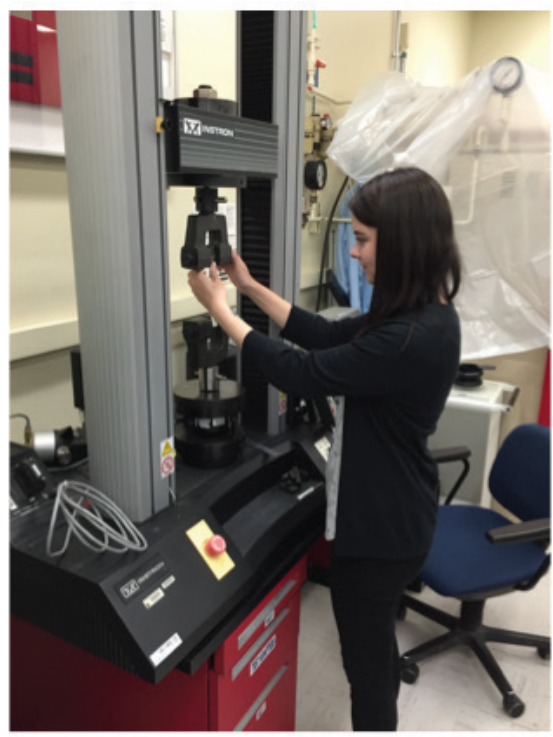

Fig. 6: The tensile testing machine used in this study is manufactured by Instron and located in the Advanced Materials Lab at JSC.

Fig. 7: Representation of the Specimen configuration for tabbed Spectra strand testing. Green squares represent cardboard tabs added to each end of Spectra strand to aid in gripping. 
Although the Spectra threads no longer slipped out of the testing apparatus grips, recorded breaking force and elongation values still varied producing a standard deviation above the desired range. Very few specimen failed in the same manner; several of the strands broke cleanly in the center, some strands contained only a few broken fibers at the maximum load, and others broke near the tab/grip (Fig. 8). Despite the discrepancies in breaking behavior, this test showed that the epoxied cardboard tab method is a successful way to prevent grip slippage when testing individual strands of Spectra.

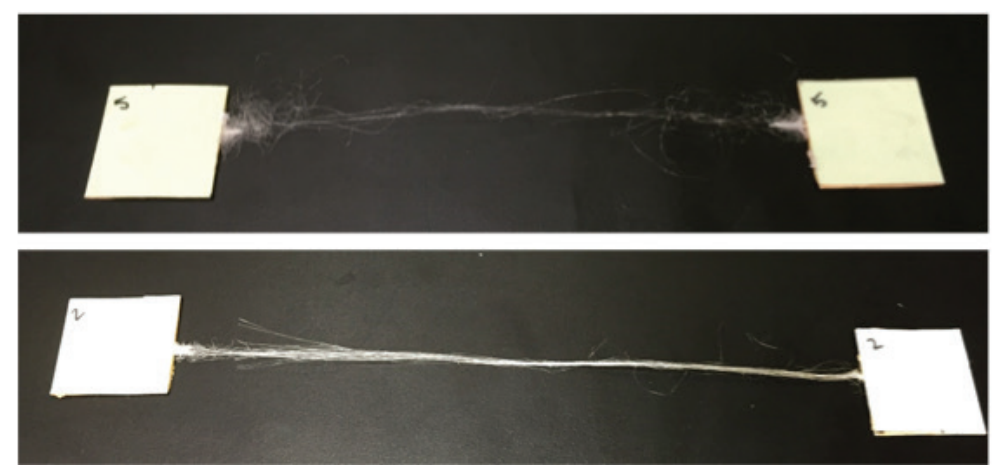

Fig. 8: Two tabbed strands of Spectra after tensile testing. Each strand is from the same sample lot (fill direction), but failed differently.

The next round of tensile testing for Spectra will again attempt the ASTM 5035 strip testing method, with the addition of epoxied cardboard tabs to the gripped area of each specimen. If the test is considered successful, the other space suit fabrics included in the study will be tested by the same method. In addition to tensile testing, future testing may include puncture testing as outlined in ASTM F1342 (Standard Test Method for Protective Clothing Material Resistance to Puncture). During my internship, I designed, procured components for, and built a fixture to be used in puncture testing. A modified version of ASTM F1342, to account for our sample size and fixture, may be performed in the future if standard tensile testing methods are unsuccessful. Once an adequate test method is decided upon, both irradiated and non-irradiated fabric samples will be tested and compared to determine if there is an effect on tensile properties of space suit fabrics due to SPE and GCR radiation.

\section{Concluding Remarks}

Overall, I have had an incredible experience as an intern with the SSA team. I was able to contribute meaningful work to several ongoing projects, and I learned a lot about the design, composition, and functionality of space suits. With the ability to work closely with several members of the SSA team, I gained experience with various space suit components and NASA processes for testing these components. In working on the irradiated materials testing project, I was introduced to and became familiar with ASTM test methods. Another important aspect of my internship was that I was able to learn about the current state of space suits and future goals in space suit development. I hope to work with space suits again in the future at JSC, and to eventually contribute directly to the development of space suits that will be used on upcoming missions to Mars. 
References

ASTM D2256-10 (Reapproved 2015), Standard Test Method for Tensile Properties of Yarns by the Single-Strand Method, American Society for Testing and Materials International, 100 Barr Harbor Drive, PO Box C700, West Conshohocken, PA 19428-2959

ASTM D5035-11 (Reapproved 2015), Standard Test Method for Breaking Force and Elongation of Textile Fabrics (Strip Method), American Society for Testing and Materials International, 100 Barr Harbor Drive, PO Box C700, West Conshohocken, PA 19428- 2959

ASTM F1342-05 (Reapproved 2013), Standard Test Method for Protective Clothing Material Resistance to Puncture, American Society for Testing and Materials International, 100 Barr Harbor Drive, PO Box C700, West Conshohocken, PA 194282959

Schimmerling, W., Ph.D. (2011, February 5). The Space Radiation Environment: An Introduction. Retrieved August 2nd, 2016, from https://three.jsc.nasa.gov/concepts/SpaceRadiationEnviron.pdf

Walsh, Sarah K. (2015, January 1). "Next Generation Life Support: High Performance EVA Glove.” From NASA Tehnical Reports Server (NTRS). Retrieved August 2nd, from http://ntrs.nasa.gov/search.jsp?R=20150014484

Weisstein, Eric W. "Correlation Coefficient.” From MathWorld--A Wolfram Web Resource. Retrieved August 2nd, 2016, from http://mathworld.wolfram.com/CorrelationCoefficient.html 